\title{
A theory of nonvertical triplet energy transfer in terms of accurate potential energy surfaces: The transfer reaction from $\pi, \pi *$ triplet donors to 1,3,5,7-cyclooctatetraene
}

\author{
Luis Manuel Frutos ${ }^{\mathrm{a}}$ and Obis Castaño \\ Departamento de Química Física, Universidad de Alcalá, 28871 Alcalá de Henares, Madrid, Spain \\ José Luis Andrés \\ Facultad de Ciencias, Universidad Nacional de Educación a Distancia (UNED), C/Colón, 114, \\ 08222 Tarrasa, Spain \\ Manuela Merchán \\ Física, Instituto de Ciencia Molecular, Universitat de València, Dr. Moliner 50, Burjassot, \\ E-46100 Valencia, Spain
}

\author{
A. Ulises Acuña \\ Instituto de Química Física “Rocasolano," C.S.I.C., Serrano 119, 28006 Madrid, Spain
}

(Received 22 July 2003; accepted 15 October 2003)

\begin{abstract}
Triplet energy transfer (TET) from aromatic donors to 1,3,5,7-cyclooctatetraene (COT) is an extreme case of "nonvertical" behavior, where the transfer rate for low-energy donors is considerably faster than that predicted for a thermally activated (Arrhenius) process. To explain the anomalous TET of COT and other molecules, a new theoretical model based on transition state theory for nonadiabatic processes is proposed here, which makes use of the adiabatic potential energy surfaces (PES) of reactants and products, as computed from high-level quantum mechanical methods, and a nonadiabatic transfer rate constant. It is shown that the rate of transfer depends on a geometrical distortion parameter $\gamma=\left(2 g^{2} / \kappa_{1}\right)^{1 / 2}$ in which $g$ stands for the norm of the energy gradient in the PES of the acceptor triplet state and $\kappa_{1}$ is a combination of vibrational force constants of the ground-state acceptor in the gradient direction. The application of the model to existing experimental data for the triplet energy transfer reaction to COT from a series of $\pi, \pi^{*}$ triplet donors, provides a detailed interpretation of the parameters that determine the transfer rate constant. In addition, the model shows that the observed decrease of the acceptor electronic excitation energy is due to thermal activation of $\mathrm{C}=\mathrm{C}$ bond stretchings and $\mathrm{C}-\mathrm{C}$ bond torsions, which collectively change the ground-state COT bent conformation $\left(D_{2 d}\right)$ toward a planar triplet state $\left(D_{8 h}\right)$. (C) 2004 American Institute of Physics. [DOI: 10.1063/1.1631418]
\end{abstract}

\section{INTRODUCTION}

Triplet photosensitization, first characterized in condensed phases by Terenin and Ermolaev, ${ }^{1}$ is a common mechanism in photoinitiated bimolecular reactions ${ }^{2,3}$ (Scheme 1), in which the electronic energy of an initially excited triplet donor $\left({ }^{3} D\right)$ is transferred to an acceptor singlet state $\left({ }^{1} A\right)$ with an observed rate constant $k_{\exp }^{\text {en }}$ :

Scheme 1:

$$
{ }^{3} D+{ }^{1} A \stackrel{k_{\exp }^{\text {en }}}{\longrightarrow}{ }^{1} D+{ }^{3} A
$$

The actual transfer of triplet energy (TET) takes place in the range of picoseconds ${ }^{4,5}$ by an electronic exchange mechanism ${ }^{2,6}$ and, therefore, close approach of the two reactants is required $(<1 \mathrm{~nm})$. When the balance between the energies of the donor and acceptor triplet states, $\Delta E_{T}=E_{T}^{D}-E_{T}^{A}$, is positive ("exothermic" TET) the ob-

${ }^{a)}$ Electronic mail: luisma.frutos@uah.es served rate in fluid solution is nearly diffusion controlled. ${ }^{2,5,7,8}$ In the endothermic case $\left(\Delta E_{T}<0\right)$ the transfer rate can still prevail over other triplet quenching channels if the energy deficit is not too large $\left(\mid \Delta E_{T}\right.$ $\mid \approx 3-4 \mathrm{kcal} / \mathrm{mol}, 1 \mathrm{kcal}=4.183 \mathrm{~kJ})$, as in a conventional thermally activated Arrhenius process. This type of kinetics was demonstrated by Sandros in a series of pioneering studies in solution, ${ }^{7}$ where the well-known expression for the rate constant of the activated transfer was introduced: $k_{\exp }^{\mathrm{en}}$ $=k^{\prime} \exp \left(-\Delta E_{T} / R T\right)$.

On the other hand, it was realized at the outset ${ }^{9}$ that for some acceptor compounds, such as cis-stilbene, the TET rate of very endothermic processes (in the above-noted sense) was considerably faster than that predicted by the Sandros equation, an anomaly associated with acceptor molecules with significant conformational flexibility. In that seminal work the term nonvertical TET was also introduced, arguing that donor-acceptor interactions result in the breakdown of Franck-Condon restrictions in such a way that the acceptor geometry changed concurrently with its excitation. This demanding interpretation has been replaced nowadays by more 
conventional "hot-band" concepts, ${ }^{2,10-12}$ in which "vertical" transitions from acceptor molecules, thermally activated along specific torsional modes, account for an observed excitation energy much lower than the "spectroscopic" $T_{1} \leftarrow S_{0}$ value. Recent developments of this idea included the consideration of more than a single potential energy curve $^{13,14}$ and, most remarkably, the definition of a rate constant distribution function associated with the geometrical deformation. ${ }^{15}$ An important difficulty in the hot-band qualitative analysis is, of course, the identification of the crucial deforming vibrations that are most effective in lowering the excitation energy.

Balzani and co-workers, ${ }^{16(a), 16(b)}$ noting the similarity between electron and triplet energy transfer reactions, developed an expression for the molecular TET rate constant $\left(k_{e}\right)$ as a function of the driving force $\Delta G^{0}\left(\equiv \Delta E_{T}\right)$ of the process, and provided a comprehensive analysis of both normal and nonvertical processes. In addition, Orlandi et al. ${ }^{16(\mathrm{c})}$ presented an alternative quantum mechanical approach, based on the treatment given by Ulstrup and Jortner for electron transfer processes, ${ }^{17}$ in which $k_{e}$ is given by the product of an electronic and a nuclear term:

$$
k_{e}=\frac{2 \pi}{\hbar} U^{2} J,
$$

where $U$ stands for the electronic exchange interaction and $J$ (the nuclear term) is the Franck-Condon weighted density of states, which normally contains the dependence on energy, temperature, and solvent properties.

This last approach has been successfully applied to a large variety of TET processes, as e.g., those giving rise to an inverted region ${ }^{18}$ and to triplet quenching through the walls of hemicarcerands. ${ }^{19}$

Very recently, Serpa et al. ${ }^{20}$ discussed in great detail different formulations of the vibrational factor $J$ for TET, based on the golden rule. On the other hand, there is no simple way of computing the $U^{2}$ value from first principles for the molecular systems of interest. ${ }^{21}$ Therefore, when a common acceptor is excited by a series of donors with "similar" structure, it is frequently assumed that the $U^{2}$ value would remain relatively constant. ${ }^{16(a), 16(b)}$ According to that, the observed variation of the rate constant has to be correlated with changes in the overlap integral $J$. Unfortunately, since the spectral data required to compute the $J$ integral are available only in a few cases, its value had to be estimated from different approximations to the true (unknown) spectra. ${ }^{20}$ In nonvertical TET processes this estimation is even more difficult, because the conformation of the (flexible) acceptor is assumed to change considerably on excitation to the triplet state and the corresponding $T_{1} \leftarrow S_{0}$ absorption would have a very low transition probability. There are a few examples ${ }^{12}$ where the expected correlation between $k_{e}$ and the vibrational factor was not observed, indicating that either the $U^{2}$ term cannot be taken as a constant in these experiments or that the approximation used to compute $J$ was too unrealistic.

Here we present an alternative analysis of the nonvertical TET processes, developed from the same "hot band" concept. The new approximation is based on the computation of accurate adiabatic potential energy surfaces (PESs) for all the states (singlet and triplet) involved in the transfer step, combined with the use of transition state theory (TST) ${ }^{22}$ of nonadiabatic processes ${ }^{23}$ to derive the rate constant for the crossing between reactant and product surfaces. It is expected that, in this way, the relationship between the energy dependency of the transfer rate and the acceptor conformational change may be better understood, and the crucial deforming molecular motions clearly identified. In addition, the utility of this approximation is illustrated by its application to the triplet energy transfer reaction of 1,3,5,7cyclooctatetraene (COT), for which accurate PESs are now available. $^{24,25}$ This cyclic, very flexible nonaromatic polyene (COT) presents the first weak absorption band at $282 \mathrm{~nm}$ in hexane solution, ${ }^{26}$ and the lowest $T_{1} \leftarrow S_{0}$ transition in the range of $65-70 \mathrm{kcal} / \mathrm{mol}$, located by electron impact spectroscopy as a broad, weakly structured band. ${ }^{27}$ A more recent estimate $^{28}$ of the energy of the elusive COT triplet state, from TET experiments in solution, yielded a value of $59 \mathrm{kcal} / \mathrm{mol}$. In contrast, a much lower value $(41 \mathrm{kcal} / \mathrm{mol})$ was derived from kinetic experiments that included a reversible transfer step. ${ }^{29}$ In the same work a long lifetime of $100 \mu$ s was assigned to ${ }^{3} \mathrm{COT}$ in cyclohexane solution, which seems difficult to conciliate with the low energy of the relaxed triplet state $\left(\sim 22 \mathrm{kcal} / \mathrm{mol}\right.$ above the $D_{2 d}$ ground state $\left.{ }^{30}\right)$ and the noted molecular flexibility of the polyene.

Nowadays, COT is used as a sort of universal triplet scavenger in organic dye lasers, because it can improve the operation of lasing dyes with triplet energies as low as 40 $\mathrm{kcal} / \mathrm{mol}{ }^{29}$ Not unexpectedly, when COT was used as acceptor for a series of triplet donors, a strong deviation from the prediction of the Sandros equation was discovered, ${ }^{28}$ extending over a large span of the energy deficit $(>20 \mathrm{kcal} / \mathrm{mol})$, that reflects the large nonvertical behavior of the cyclic polyene. This exceptional deviation was explained as due to torsions around the annulene single bonds, which might largely decrease the energy required to populate the triplet state. ${ }^{28}$

The interpretation of the experimental TET data of COT requires an expression for the transfer rate constant as a function of the triplet energy of a series of donor molecules. This expression is derived here from the following sequence. In a first step we introduce the adiabatic PES of the reactant and product collision complex; a gradient difference vector is defined, that yields the difference between the energy gradient in each of the two PESs. The interaction leading to surface crossing and triplet energy transfer is usually explained as due to electron exchange (Dexter mechanism ${ }^{6}$ ) and depends on the proximity between donor and acceptor molecules. Consequently, we next define an electron coupling vector, which yields the extent of this interaction. These two vectors are assigned to well-defined nuclear coordinates of the encounter complex. In a second step, the rate constant for the crossing reaction is obtained, based on the nonadiabatic formulation of transition-state theory. Finally, the activation energy of the transfer process is derived as a function of the donor triplet energy for the case of nonvertical behavior. 
a)

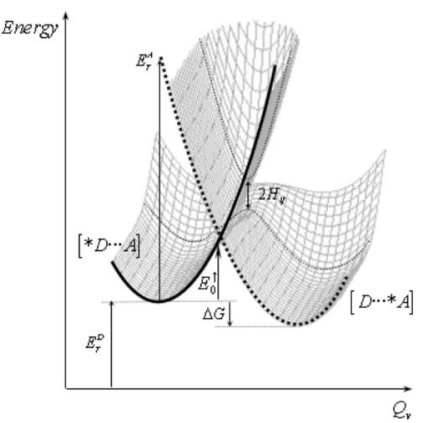

b)

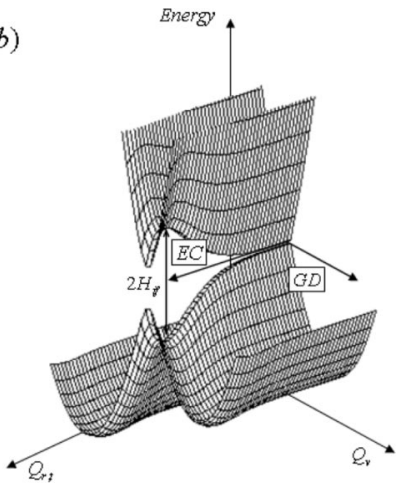

FIG. 1. The triplet energy transfer reaction as pictured by the crossing between the potential energy surfaces (PES) of the donor-acceptor $[D \cdots A]$ collision complex. (a) The potential energy surface as a function of the molecular vibrational coordinates $\left(Q_{\nu}\right) . E_{T}^{D}$ and $E_{T}^{A}$ are the (optical) excitation energies of the donor and acceptor triplet states, respectively; $E_{0}^{\dagger}$ is the energy at the crossing point of the adiabatic PES; $H_{i f}$ represents the amplitude (exaggerated) of the electron exchange interaction and $\Delta G$ the free energy of the process. (b) Another perspective of the PES, now as a function of the donor-acceptor relative position, given by the $Q_{r, t}$ coordinates. The arrows labeled as EC and GD show the direction of electronic coupling and energy gradient difference vectors, respectively. The avoided crossing due to electron exchange coupling perturbation $\left(H_{i f}\right)$ results in the lowering of the energy barrier between reactants $\left[{ }^{*} D \cdots A\right]$ and products $[D \cdots * A]$, compared with that in the adiabatic PES.

\section{RESULTS AND DISCUSSION}

\section{A. The potential energy surfaces of the triplet energy transfer reaction}

The transfer reaction (Scheme 1) can be formally expanded ${ }^{16}$ to separate the diffusive $\left(k_{d}, k_{-d},\right)$ and energy transfer steps $\left(k_{e}, k_{-e}\right)$ as:

Scheme 2:

$$
{ }^{3} D+{ }^{1} A \underset{k_{-d}}{\stackrel{k_{d}}{\rightleftarrows}}\left[{ }^{3} D \cdots{ }^{1} A\right] \underset{k_{-e}}{\stackrel{k_{e}}{\rightleftarrows}}\left[{ }^{1} D \cdots{ }^{3} A\right] \underset{k_{d}}{\stackrel{k_{-d}}{\rightleftarrows}} D+{ }^{3} A
$$

The transfer process involves only the PES of reactant $\left[{ }^{3} D \cdots{ }^{1} A\right]$ and product $\left[{ }^{1} D \cdots{ }^{3} A\right]$ encounter complexes, which can be considered as a supermolecule in two different electronic states [Fig. 1(a)] (in the following the more convenient $\left[{ }^{*} D \cdots A\right][D \cdots * A]$ symbols will be used). The energy transfer process consists on the passage of the system from the first to the second state. It is assumed here that long-lived exciplexes are not formed in the TET reaction in solution, ${ }^{11}$ due to the weak donor-acceptor interaction. If this interaction is negligible, the relative energy of each complex would be given by $E[* D \cdots A]=E(* D)+E(A)$ and $E[D \cdots * A]=E(D)+E(* A)$. However, when the two PESs are near isoenergetic some sort of interaction must be present for the crossing to occur, and a coupling correction to the energy should be introduced.

The PES of each collision complex is a space function of internal coordinates only, with $F$ degrees of freedom, given by $F=3\left(N_{D}+N_{A}\right)-6$, where $N_{D(A)}$ is the number of atoms of the donor (acceptor), each with its associated coordinate. The total number of coordinates, $Q$, is separated here in two groups, $Q=\left\{Q_{r, t}, Q_{\nu}\right\}$, where $Q_{r, t}$ defines the relative posi- tion of donor and acceptor (three coordinates for translation and three for rotation) and $Q_{\nu}$ corresponds to all molecular vibrations $\left[3\left(N_{D}+N_{A}\right)-12\right]$. In the transfer step, the spin and spatial symmetry of the total wave function is preserved, resulting in an avoided crossing in the regions approaching energy degeneracy ${ }^{31}$ [Fig. 1(b)]. The two special dimensions where the energy degeneracy disappears are defined by the electronic coupling $\left(\mathbf{v}_{\mathrm{EC}}\right)$ and gradient difference $\left(\mathbf{v}_{\mathrm{GD}}\right)$ vectors. The first one (EC) indicates the maximum coupling strength, while the second (GD) gives the difference between the energy gradient vectors of the PES of two complexes at a given nuclear configuration: $[* D \cdots A]$ and $[D \cdots * A]$ (vide infra). In the following paragraphs each vector is assigned to a well-defined subspace of coordinates.

The electron exchange between donor and acceptor is expressed by the coupling matrix term $H_{i f}=\left\langle\Psi_{\left[{ }^{*} \cdots A\right]}\left|\hat{H}^{\prime}\right| \Psi_{[D \cdots A *]}\right\rangle \quad(i=$ initial, $f=$ final $) \quad$ in which

$$
\hat{H}^{\prime}=\sum_{i, j} \frac{1}{r_{i j}}
$$

is the electronic coupling Hamiltonian, where the indices $i, j$ denote the donor and acceptor electrons, respectively. In a first-order approximation, the wave functions can be taken as $\Psi_{\left[*_{D} \cdots A\right]}=\wp \psi_{* D} \psi_{A}$, and $\Psi_{[D \cdots * A]}=\wp \psi_{D} \psi_{*_{A}}$ where $\psi$ stands for the electronic wave function of the isolated molecules, and $\wp$ is a permutation operator which acts antisymmetrizing the wave function product under the permutation between $i$ and $j$ electrons. ${ }^{6(c)}$ The molecular wave functions, in a first approximation, can be taken as those of the highest occupied molecular orbital (HOMO) and lowest unoccupied molecular orbital (LUMO) of both the singlet and the triplet states of the reacting molecules. ${ }^{6(\mathrm{~b})}$ According to that, $H_{i f}$ is a function only of the relative position of the donor and acceptor, given by the $Q_{r, t}$ coordinates, because the change on the electronic orbital overlap due to internal vibrations is expected to be comparatively much less important. Therefore, the electronic coupling vector $\left(\mathbf{v}_{\mathrm{EC}}\right)$ would depend only on the relative position of donor and acceptor, that is, on the $Q_{r, t}$ coordinates. Thus, for a given nuclear configuration $\mathbf{Q}$ the electronic coupling vector is given by

$$
\mathbf{v}_{\mathrm{EC}}=\sum_{\mu=1}^{6} \mathbf{u}_{\mu}\left(\frac{\partial H_{i f}\left(Q_{r, t}\right)}{\partial q_{\mu}}\right)_{\mathbf{Q}}, \quad q_{\mu} \in Q_{r, t},
$$

where $\mathbf{u}_{\mu}$ is the corresponding unit vector and $H_{i f}$ the coupling energy between initial and final states.

It could also be possible to include an additional vibronic (derivative) coupling term into the Hamiltonian, because of the eventual near-degeneration of the PESs of the two electronic states. However, the contribution of the vibronic coupling to the electronic energy would be only a small fraction of the electronic one. This can be shown in a qualitative way as follows:

In terms of perturbation theory, the vibronic coupling term can be formulated as 


$$
\begin{aligned}
W_{i f}= & \left\langle\Psi_{[* D \cdots A]}\left|\sum_{i=r, t, \nu} \frac{\partial}{\partial Q_{i}}\right| \Psi_{[D \cdots A}\right\rangle \\
\approx & \mid\left\langle\Psi_{[* D \cdots A]}\left|\sum_{i=r, t, \nu} \frac{\partial\left(H_{D}^{0}+H_{A}^{0}+H^{\prime}\right)}{\partial Q_{i}}\right|\right. \\
& \left.\times \Psi_{[D \cdots A *]}\right\rangle \mid,
\end{aligned}
$$

where $H_{A, D}^{0}$ is the electronic Hamiltonian of the isolated molecule and $H^{\prime}$ is the electronic coupling defined earlier. If the internal conversion rate in each isolated molecule is negligible (otherwise the triplet energy transfer process would not occur), the first two terms in the sum of Eq. (3) vanish, that is

$$
\left\langle\Psi_{\left[*_{D} \cdots A\right]}\left|\sum_{i=r, t, \nu} \frac{\partial\left(H_{D, A}^{0}\right)}{\partial Q_{i}}\right| \Psi_{[D \cdots A *]}\right\rangle=0,
$$

and the resultant expression would be

$$
\left.W_{i f} \approx \mid\left\langle\Psi_{\left[*_{D} \cdots A\right]}\left|\sum_{i, j} \frac{\partial r_{i j}^{-1}}{\partial Q_{r, t}}\right| \Psi_{[D \cdots A}\right\rangle\right] \mid .
$$

Taking $r_{i j}=R$ as a constant (the average distance between electrons) Eq. (4) can be further simplified to give

$$
\begin{aligned}
W_{i f} & \approx\left|\left\langle\Psi_{\left[*_{D} \cdots A\right]}\left|\frac{\partial R^{-1}}{\partial Q_{r, t}}\right| \Psi_{[D \cdots A}\right\rangle\right| \\
& \left.=\mid R^{-2}\left\langle\Psi_{\left[{ }^{*} D A\right]} \mid \Psi_{[D \cdots A}\right\rangle\right] \mid \\
& =R^{-2} S_{i f}\left(Q_{r, t}\right),
\end{aligned}
$$

where $S_{i f}$ represents the electron orbital overlap as a function of the donor-acceptor relative position coordinates, $Q_{r, t}$. Since in the present approximation the dependence of the electronic coupling on electron separation $(R)$ is given by $H_{i f} \approx R^{-1} S_{i f}$, the contribution of the vibronic term $W_{i f}$ to the total electronic energy would be very small for the same value of the electron separation. In summary, the coupling interaction between reactants and products PES arise essentially from electron exchange, with a small contribution from vibronic coupling. The components of the vector associated with this interaction $\left(\mathbf{v}_{\mathrm{EC}}\right)$ extend only over rotational and translational coordinates $\left(Q_{r, t}\right)$ describing the proximity of donor/acceptor within the collision complex.

On the other hand, the energy gradient vector for the two encounter complexes and, therefore, the energy gradient difference vector depends only on internal (vibrational) coordinates, because the donor-acceptor interaction in the transfer process is so weak that no new bonds are formed (that might store energy). Accordingly, the energy gradient difference would not depend significantly on the donor-acceptor relative position:

$\left(\frac{\partial\left(E_{\left[*_{D} \cdots A\right]}-E_{[D \cdots A}\right)}{\partial q_{\mu}}\right)_{\mathbf{Q}} \approx 0, \quad q_{\mu} \in\left\{Q_{r, t}\right\}$,

for any configuration $\mathbf{Q}$.

Hence, the energy gradient difference vector for a configuration $\mathbf{Q}$ can be defined as
$\mathbf{v}_{\mathrm{GD}}=\sum_{\mu=1}^{N} \mathbf{u}_{\mu}\left(\frac{\partial\left(E_{[* D \cdots A]}-E_{[D \cdots A}\right)}{\partial q_{\mu}}\right)_{\mathbf{Q}}, \quad q_{\mu} \in Q_{\nu}$.

In conclusion, the $\mathbf{v}_{\mathrm{EC}}$ vector is associated with the $Q_{r, t}$ subset of coordinates, while $\mathbf{v}_{\mathrm{GD}}$ is associated with the vibrational subset $Q_{\nu}$.

\section{B. The energy transfer rate constant}

The rate constant for the crossing between reactants and products energy surfaces can be expressed from transition state theory ${ }^{22}$ as

$$
k_{e}=\chi \frac{f^{\neq}}{f_{1} f_{2} \cdots} \frac{k_{B} T}{h} \exp \left(-\frac{V_{0}}{k_{B} T}\right),
$$

where $f^{\neq}$is the partition function of the activated complex and $f_{i}$ the partition functions of reactants and products, $V_{0}$ is the activated complex energy, $\chi$ the transmission coefficient, and the other symbols have the usual meaning.

The crossing step can be described as a nonadiabatic transition in a weakly coupled system. ${ }^{32}$ In that case, the value of $\chi$ is well described by ${ }^{23}$

$$
\chi \simeq \frac{4 \pi H_{i f}^{2}}{\hbar v\left|s_{f}-s_{i}\right|},
$$

where $v$ is the relative velocity with which the system passes the point of closest approach and $\left|s_{f}-s_{i}\right|$ is the absolute magnitude of the difference between the energy gradient of the two crossing surfaces. The weakly allowed nonadiabatic crossing results in a lower energy barrier compared with that corresponding to the intersection of adiabatic surfaces $\left(E_{0}^{\dagger}\right)$, that is: $V_{0}=E_{0}^{\dagger}-H_{i f}$ [Fig. 1(b)]. Accordingly, the rate constant for nonadiabatic crossing becomes

$$
k_{e}=\frac{4 \pi H_{i f}^{2}}{\hbar v\left|s_{f}-s_{i}\right|} \frac{f^{\neq}}{f_{1} f_{2} \cdots} \frac{k T}{h} \exp \left(\frac{H_{i f}}{k_{B} T}\right) \exp \left(-\frac{E_{0}^{\dagger}}{k_{B} T}\right),
$$

where $E_{0}^{\dagger}$ is the activated complex energy that would be obtained from adiabatic PES. As noted earlier, the value of $E_{0}^{\dagger}$ does not depend on the donor-acceptor separation within the collision complex, while that of $H_{i f}$ changes very rapidly (exponentially) with that distance. Moreover, since $H_{i f}<k_{B} T$, the exponential term $e^{H_{i f} / k_{B} T} \approx 1$ and the rate constant given by Eq. (9) shows the exponential dependence with the donor-acceptor distance observed in simple systems. ${ }^{6(\mathrm{~b}), 33}$

\section{The energy of the activated complex}

In this section the value of the energy of the activated complex would be derived, as a function of the triplet energy of the donor. The coupling term $H_{i f}$ [Eq. (9)] depends only on the orbital overlap and, therefore, it is independent of the donor energy. According to that, one only needs to determine the dependence of $E_{0}^{\dagger}$ (the adiabatic component of the activation energy) on the triplet donor energy. For moderate temperatures, the potential energy of the complex in the initial state, $[* D \cdots A]$, defined as $E_{0}(Q) \equiv E_{\left[*^{*} \cdots A\right]}\left(Q_{\nu}\right)$, is well 
represented classically as a function of electronic energies $E_{\text {elec }}^{A}{ }^{*} D$, vibrational coordinates $\left(q_{\mu, \nu}^{A, D}\right)$, and harmonic force constants $\left(\kappa_{\mu \nu}^{A,{ }^{*} D}\right)$ by

$$
\begin{aligned}
E_{0}(Q)= & E_{0}\left(q_{1}^{A}, q_{2}^{A}, \ldots, q_{3 N_{A}-6}^{A}, q_{1}^{D}, q_{2}^{D}, \ldots, q_{3 N_{D}-6}^{D}\right) \\
= & E_{\mathrm{elec}}^{A}+E_{\mathrm{elec}}^{* D}+\frac{1}{2} \sum_{\mu}^{3 N_{A}-6} \sum_{\nu}^{3 N_{A}-6} \kappa_{\mu \nu}^{A} q_{\mu}^{A} q_{\nu}^{A} \\
& +\frac{1}{2} \sum_{\mu}^{3 N_{D}-6} \sum_{\nu}^{3 N_{D}-6} \kappa_{\mu \nu}^{* D} q_{\mu}^{D} q_{\nu}^{D}+\cdots
\end{aligned}
$$

where

$$
\kappa_{\mu \nu}^{A, * D}=\left(\frac{\partial^{2} E_{0}}{\partial q_{\mu}^{D, A} \partial q_{\nu}^{D, A}}\right)_{e} .
$$

The $e$ subscript is used here to identify the equilibrium configuration of the $\left[{ }^{*} D \cdots A\right]$ complex. As noted earlier, due to the very weak coupling there is no accumulation of energy in the other subset of degrees of freedom $Q_{r, t}$.

Similarly, the potential energy surface of the complex in the final state, $\left[D \cdots A^{*}\right]$, defined as $E_{1}(Q)$ $\equiv E_{[D \cdots A *]}\left(Q_{\nu}\right)$, can be expressed by

$$
\begin{aligned}
E_{1}(Q)= & E_{1}\left(q_{1}^{A}, q_{2}^{A}, \ldots, q_{3 N_{A}-6}^{A}, q_{1}^{D}, q_{2}^{D}, \ldots, q_{3 N_{D}-6}^{D}\right) \\
= & E_{\mathrm{elec}}^{*_{A}}+E_{\mathrm{elec}}^{D}+\sum_{\mu=1}^{3 N_{A}-6} \alpha_{\mu}^{* A} q_{\mu}^{A}+\sum_{\mu=1}^{3 N_{D}-6} \alpha_{\mu}^{D} q_{\mu}^{D} \\
& +\frac{1}{2} \sum_{\mu}^{3 N_{A}-6} \sum_{\nu}^{3 N_{A}-6} \kappa_{\mu \nu}^{* A} q_{\mu}^{A} q_{\nu}^{A} \\
& +\frac{1}{2} \sum_{\mu}^{3 N_{D}-6} \sum_{\nu}^{3 N_{D}-6} \kappa_{\mu \nu}^{D} q_{\mu}^{D} q_{\nu}^{D}+\cdots
\end{aligned}
$$

where

$$
\begin{aligned}
& \alpha_{\mu}^{*_{A}}=\left(\frac{\partial E_{1}}{\partial q_{\mu}^{A}}\right)_{e}, \quad \alpha_{\mu}^{D}=\left(\frac{\partial E_{1}}{\partial q_{\mu}^{D}}\right)_{e}, \\
& \kappa_{\mu \nu}^{*_{A}, D}=\left(\frac{\partial^{2} E_{1}}{\partial q_{\mu}^{A, D} \partial q_{\nu}^{A, D}}\right)_{e} .
\end{aligned}
$$

Equation (11) corresponds to expansion of the $\left[D \cdots A^{*}\right]$ PES up to the quadratic terms, using the same origin and set of coordinates as those of Eq. (10).

In the crossing space $E_{0}(Q)=E_{1}(Q)$ and, therefore, from Eqs. (7) and (8) we have

$$
\begin{aligned}
E_{T}^{D}-E_{T}^{A}= & \sum_{\mu=1}^{3 N_{A}-6} \alpha_{\mu}^{* A} q_{\mu}^{A}+\sum_{\mu=1}^{3 N_{D}-6} \alpha_{\mu}^{D} q_{\mu}^{D} \\
& +\frac{1}{2} \sum_{\mu=1}^{3 N_{A}-6} \sum_{\nu=1}^{3 N_{A}-6}\left(\kappa_{\mu \nu}^{* A}-\kappa_{\mu \nu}^{A}\right) q_{\mu}^{A} q_{\nu}^{A} \\
& +\frac{1}{2} \sum_{\mu=1}^{3 N_{D}-6} \sum_{\nu=1}^{3 N_{D}-6}\left(\kappa_{\mu \nu}^{D}-\kappa_{\mu \nu}{ }^{D}\right) q_{\mu}^{D} q_{\nu}^{D}+\cdots,
\end{aligned}
$$

where

$$
E_{T}^{A} \equiv\left(E_{\text {elec }}^{* A}-E_{\text {elec }}^{A}\right) \text { and } E_{T}^{D} \equiv\left(E_{\text {elec }}^{* D}-E_{\text {elec }}^{D}\right) .
$$

In those cases where the first, linear term provides the largest contribution to the energy difference, Eq. (12) can be greatly simplified to yield

$$
E_{T}^{D}-E_{T}^{A} \approx \sum_{\mu=1}^{3 N_{A}-6} \alpha_{\mu}^{{ }^{*} A} q_{\mu}^{A}+\sum_{\mu=1}^{3 N_{D}-6} \alpha_{\mu}^{D} q_{\mu}^{D} .
$$

This expression shows that the energy difference between donor and acceptor triplet states at the crossing region of the PES is matched by thermal activation of the internal degrees of freedom of the two reactants. It is clear that Eq. (13) would be more accurate the larger the value of the $\alpha_{\mu, \nu}^{* A, D}$ coefficients. In fact, these coefficients are the components of the energy gradient vector in the complex final state after a vertical excitation; a large gradient value (i.e., large $\alpha$ coefficients) indicates an important change in the conformation of $D, A$ or both in the transfer process. This is precisely one of the characteristics of nonvertical processes, as discussed earlier, in which the deviation from the "normal" TET was associated with a flexible acceptor structure. ${ }^{34}$ On the other hand, it should be noted that a large conformational change does not imply necessarily a large energy gradient.

Another distinctive property of the acceptor singlet state in nonvertical TET reactions is that small changes in vibrational coordinates $\left(q_{i}\right)$ result in large changes on the energy deficit $E_{T}^{A}-E_{T}^{D}$, which also contributes to the accuracy of the approximation in Eq. (13).

The triplet energy transfer processes of interest here are those involving flexible acceptor molecules (as, e.g., cisstilbene and COT). Therefore, Eq. (13), can be further simplified for those cases in which the contribution of the donor deformation to the change in the excitation energy is negligible, to yield

$$
E_{T}^{D}-E_{T}^{A}=\sum_{\mu=1}^{3 N_{A}-6} \alpha_{\mu}^{* A} q_{\mu}^{A}
$$

Finally, Eq. (14) can be recast in a new orthonormal basis set, in which the gradient vector for the vertical excitation of $* A$ is one of the basis vectors, to give:

$$
E_{T}^{A}-E_{T}^{D}=|\mathbf{g}| \xi_{1}^{A},
$$

where

$$
|\mathbf{g}|=\left|\nabla\left[E_{1}(Q)\right]\right|=\left\{\sum_{\mu=1}^{3 N_{A}-6}\left(\alpha_{\mu}^{* A}\right)^{2}\right\}^{1 / 2}
$$

and $\xi_{1}^{A}$ is the coordinate along the gradient vector.

In the above expression $|\mathbf{g}|(\equiv g)$ is the norm of the gradient difference vector $\left(\mathbf{v}_{\mathrm{GD}}\right)$ between the gradient of the $T_{1}$ and $S_{0}$ surfaces, i.e., $\mathbf{g}=\mathbf{g}\left(T_{1}\right)-\mathbf{g}\left(S_{0}\right)$. Since the excitation of the acceptor $S_{0}$ state takes place from a configuration close to the equilibrium position where the gradient is negligible, the $\mathbf{g}$ value corresponds essentially to the gradient of the triplet state surface, only $\left[\mathbf{g} \simeq \mathbf{g}\left(T_{1}\right) \equiv \mathbf{g}_{1}\right]$. According to 
this, the acceptor excitation energy in the new basis set depends only on a single coordinate $\xi_{1}^{A}$, which is that of the triplet acceptor gradient vector:

$$
E_{T}^{A}-E_{T}^{D}=g_{1} \xi_{1}^{A} .
$$

This can be considered as the generalization of the above-discussed "hot-band" concept, because, as shown by Eq. (16), the energy deficit can be lessened by geometrical distortions of the ground state acceptor along vibrational coordinates $\left(\xi_{1}^{A}\right)$ that correspond to a large change in the downward slope of the triplet potential energy surface. Distortions in other directions are orthogonal vectors to the gradient $\mathbf{g}$ and, therefore, do not contribute, in this formalism, to the energy expansion in Eq. (15). In addition, it becomes clear that the whole set of components of the gradient vector (bond stretchings, torsions, and bendings) is the important factor in determining the acceptor excitation energy.

The vibrational energy $\left(E_{0, v i b}\right)$ of the initial complex $\left[{ }^{*} D \cdots A\right]$ can be obtained from Eq. (10) by subtracting from the total energy the electronic energy, to give

$$
\begin{aligned}
E_{0, \mathrm{vib}}= & \frac{1}{2} \sum_{\mu}^{3 N_{A}-6} \sum_{\nu}^{3 N_{A}-6} \kappa_{\mu \nu}^{A} q_{\mu}^{A} q_{\nu}^{A} \\
& +\frac{1}{2} \sum_{\mu}^{3 N_{D}-6} \sum_{\nu}^{3 N_{D}-6} \kappa_{\mu \nu}^{* D} q_{\mu}^{D} q_{\nu}^{D}+\cdots .
\end{aligned}
$$

This expression can also be redefined in a new basis set, in which the coordinates are $\xi_{1}^{A}$ and those, for the acceptor only, diagonalizing the Hessian matrix of the energy in the subspace of coordinates orthogonal to $\xi_{1}^{A}$, that is, $\xi_{i}^{A}$ with $i=2, \ldots, 3 N_{A}-6$. If the donor coordinates $q_{i}^{D}$ are preserved, we have

$$
\begin{aligned}
E_{0, \mathrm{vib}}= & \frac{1}{2} \sum_{i}^{3 N_{A}-6} \sum_{j}^{3 N_{A}-6} \kappa_{i j}^{A} \xi_{i}^{A} \xi_{j}^{A} \\
& +\frac{1}{2} \sum_{\mu}^{3 N_{D}-6} \sum_{\nu}^{3 N_{D}-6} \kappa_{\mu \nu}^{* D} q_{\mu}^{D} q_{\nu}^{D} \\
= & \frac{1}{2} \kappa_{11}^{A}\left(\xi_{1}^{A}\right)^{2}+\sum_{i=2}^{3 N_{A}-6} \kappa_{i 1}^{A} \xi_{i}^{A} \xi_{1}^{A} \\
& +\frac{1}{2} \sum_{i=2}^{3 N_{A}-6} \kappa_{i i}^{A}\left(\xi_{i}^{A}\right)^{2}+\frac{1}{2} \sum_{\mu}^{3 N_{D}-6} \sum_{\nu}^{3 N_{D}-6} \kappa_{\mu \nu}^{* D} q_{\mu}^{D} q_{\nu}^{D},
\end{aligned}
$$

where

$$
\kappa_{i j}^{A}=\left(\frac{\partial^{2} E_{0}}{\partial \xi_{i}^{A} \partial \xi_{j}^{A}}\right)_{0} .
$$

The acceptor vibrational energy, $E_{0 \text {,vib }}^{\dagger}$, corresponding to the configuration of the minimum energy in the crossing subspace (i.e., the activation energy) and consistent with Eq. (16), can be found by defining the Lagrangian function $(L)$ as

$$
L=E_{0, \mathrm{vib}}+\lambda\left(E_{T}^{A}-E_{T}^{D}-g \xi_{1}^{A}\right) .
$$

Differentiating relative to $\xi_{1}^{A}$ and $\xi_{n}^{A}$ gives

$$
\begin{aligned}
& \frac{\partial L}{\partial \xi_{1}^{A}}=\kappa_{11}^{A} \xi_{1}^{A}+\sum_{i=2}^{3 N_{A}-6} \kappa_{i 1}^{A} \xi_{i}^{A}+\lambda g=0, \\
& \frac{\partial L}{\partial \xi_{n}^{A}}=\kappa_{1 n}^{A} \xi_{1}^{A}+\kappa_{n n}^{A} \xi_{n}^{A}=0 \Rightarrow \xi_{n}^{A}=-\frac{\kappa_{1 n}^{A}}{\kappa_{n n}^{A}} \xi_{1}^{A} .
\end{aligned}
$$

Finally, by replacing in Eq. (18) the value given by Eqs. (16) and (20), the following expression results:

$$
\begin{aligned}
E_{0, \mathrm{vib}}^{\dagger} & =\frac{1}{2} \frac{\kappa_{11}^{A}-\sum_{i=2}^{3 N_{A}-6} \frac{\left(\kappa_{i 1}^{A}\right)^{2}}{\kappa_{i i}^{A}}}{g^{2}}\left(E_{T}^{A}-E_{T}^{D}\right)^{2} \\
& \equiv \frac{1}{2} \frac{\kappa_{1}}{g^{2}}\left(E_{T}^{A}-E_{T}^{D}\right)^{2} .
\end{aligned}
$$

It should be recalled that the minimum energy at the crossing point is independent of the donor coordinates, because the energy change (decrease) of the system depends on the acceptor coordinates $\xi_{1}^{A}$ [Eq. (16)].

This important relationship [Eq. (21)] gives the value of the energy barrier for the direct energy transfer reaction as a function of the force constants of ground-state vibrations $\left(\kappa_{1}\right)$ for the acceptor, the energy gradient on the triplet-state surface of the acceptor molecule $(g)$, and the difference between the optical excitation energies of the acceptor and donor triplet states. Since the PESs as defined here do not include any contribution from solvent effects, the energy barrier corresponds to a solvent-free environment or to one in which the solvent is unperturbed. This is, of course, an oversimplification but it is not expected to result in large errors in the computed activation energy, since TET does not involve a major redistribution of charges. On the other hand, the value of the preexponential factor in Eq. (9) may be sensitive to solvent properties, due to the corresponding changes in the velocity term $v$.

By substituting Eq. (21) into the expression for the transfer rate constant we have

$$
\begin{aligned}
k_{e}= & \frac{4 \pi H_{i f}^{2}}{\hbar v\left|s_{f}-s_{i}\right|} \frac{f^{\neq}}{f_{1} f_{2} \cdots} \frac{k_{B} T}{h} \exp \left[-\left(\frac{E_{0, \mathrm{vib}}^{\dagger}}{k_{B} T} \frac{H_{i f}}{k_{B} T}\right)\right] \\
= & \frac{4 \pi H_{i f}^{2}}{\hbar v\left|s_{f}-s_{i}\right|} \frac{f^{\neq}}{f_{1} f_{2} \cdots} \frac{k_{B} T}{h} \exp \left(\frac{H_{i f}}{k_{B} T}\right) \\
& \times \exp \left[-\frac{1}{2 k_{B} T} \frac{\kappa_{1}}{g^{2}}\left(E_{T}^{A}-E_{T}^{D}\right)^{2}\right] .
\end{aligned}
$$

This expression applies for a triplet-triplet transfer reaction in which the acceptor singlet-state conformation changes substantially on excitation, compared with that of the donor molecule. The preexponential factor in Eq. (22) can now be analyzed in detail based on the properties of the abovediscussed PES. Thus, the relative velocity $v$ with which the complex crosses the coupling space would be determined by the vibrational frequency of the modes contained in $\kappa_{1}$. Since in the model discussed here the GD vector depends only on the properties of the acceptor PES (or, in other words, the decrease in excitation energy is due only to the acceptor deformation), the value of $v$ would depend on the 
acceptor vibrational frequencies, as well as on the effect of the solvent vibrational modes coupled to that of the supermolecule and temperature.

On the other hand, the parameter $\left|s_{f}-s_{i}\right|$ is just the module of the GD vector at the crossing space. In a series of experiments where the same acceptor molecule is used, this term remains constant because, as noted earlier, the gradient of the excited state is much larger than that of the $S_{0}$. Hence, $\left|s_{f}-s_{i}\right| \approx\left|s_{f}\right|=$ constant.

The electronic coupling term $H_{i f}$ is a two-center, twoelectron integral that depends mainly on the structure of both the HOMO and LUMO of the donor-acceptor pair. However, in a TET experiment involving a common acceptor and a series of donors of the same electronic type $\left(\pi, \pi^{*}\right.$, for example) in which the spatial distribution of the MOs can be taken as relatively unchanged, the $H_{i f}$ value would not be expected to depart substantially from an average value characteristic of the whole donor series. A similar reasoning can be applied to the partition functions in Eq. (22), by noting that these functions can be expressed by two factors, which depend on the degrees of freedom of the donor and acceptor, respectively. Thus, if the conformation of the selected donor molecules does not change substantially in the $T_{1} \rightarrow S_{0}$ transition, the expression for the transfer rate constant to a common acceptor may be approximated by

$$
\begin{aligned}
k_{e} & =H_{i f}^{2} k(T) \exp \left(\frac{H_{i f}}{k_{B} T}\right) \exp \left(\frac{1}{2 k_{B} T} \frac{\kappa_{1}}{g^{2}}\left(E_{T}^{A}-E_{T}^{D}\right)^{2}\right) \\
& \simeq k_{0}^{e}(T) \exp \left(-\frac{1}{2 k_{B} T} \frac{\kappa_{1}}{g^{2}}\left(E_{T}^{A}-E_{T}^{D}\right)^{2}\right) .
\end{aligned}
$$

In summary, the expression for the rate constant, as derived above, for a triplet-triplet transfer reaction between a series of rigid triplet donors and a flexible acceptor contains (i) a preexponential factor $k_{0}^{e}$ associated with the donoracceptor orbital overlap through $H_{i f}^{2} e^{H_{i f} / k_{B} T}$ and other factors, which are not expected to depend on the triplet energy difference, as noted before, ${ }^{12,16,35}$ and (ii) an exponential term with a quadratic dependence on the energy difference between the donor and the acceptor triplet states.

In Eq. (23) we may define a geometrical distortion parameter $\gamma=\left(2 g^{2} / \kappa_{1}\right)^{1 / 2}$, which would eventually determine the extent on nonvertical behavior. For acceptor molecules with large values of the energy gradient on the triplet surface, the easy activation of ground-state vibrational modes (low force constants $\kappa_{1}$ ) along the gradient direction would be very effective in reducing the triplet excitation energy (high $\gamma$ value), resulting in a large rate constant even for endothermic reactions. However, the occurrence of significant conformational changes can also be completely ineffective in this regard, unless they give rise to a large change in the tripletstate energy gradient. Moreover, since the values of $\gamma$ and $E_{T}^{A}$ are fixed for a specific acceptor molecule, Eq. (23) predicts an "inverted" region, where an increase in the donor energy available for excitation would eventually result in a lower transfer rate, ${ }^{18,19(a), 20}$ in common with the Marcus theory of electron transfer. ${ }^{36}$ However, this effect would be difficult to observe in TET reactions, due to the presence of higher energy triplets and alternative reactive channels. ${ }^{37,38}$

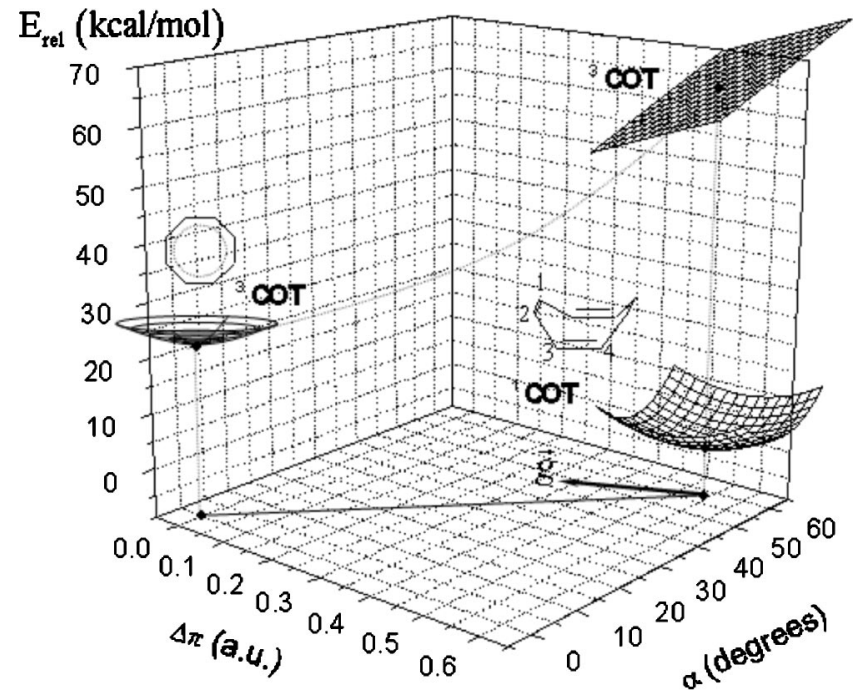

FIG. 2. The regions of the potential energy surfaces of cyclooctatetraene (COT) singlet and triplet states which are relevant for the energy transfer process, computed as described in the main text. The PES are show here as a function of the dihedral angle, $\alpha$, of the four consecutive carbon atoms (i.e., 1-2-3-4), which gives the degree of the planarity of the molecule, and the change in bond distances $\Delta \pi=d_{\mathrm{C}-\mathrm{C}}-d_{\mathrm{C}=\mathrm{C}}$, where $d_{\mathrm{C}-\mathrm{C}}$ and $d_{\mathrm{C}=\mathrm{C}}$ are the sum over all simple and double bond distances (i.e., 2-3, and 1-2), respectively. Note that the extent of $\pi$-charge delocalization is proportional to the inverse of $\Delta \pi$. The arrow indicates the direction of the energy gradient vector $\left(\mathbf{v}_{\mathrm{GD}}\right)$. This vector, which defines the relaxation of the initially excited triplet, has large component in both the $\pi$-delocalization and flattening coordinates of the molecule. The $0-0$ energy of ${ }^{3} \mathrm{COT}\left(D_{8 h}\right)$ was taken from Ref. 30.

The expression for the TET rate constant [Eq. (23)] is amenable to experimental test, because the $\gamma$ values of many molecules of interest can be computed with high accuracy by current quantum mechanical methods, as shown in the following for COT. It is important to remark that a computation of the complete PES for the singlet and triplet states is not necessary in this approximation, as only that part of the ${ }^{3} \mathrm{~A}$ surface in the vicinity of the equilibrium configuration of ${ }^{1} A$ is required (Fig. 2).

Finally, it may be instructive to compare the TST rate constant expression given by Eq. (23) with that derived from the Fermi golden rule [Eq. (1)]. In both cases, the preexponential factor depends on the square of the electronic coupling $\left(H_{i f}^{2}\right)$ between the intervening states. In addition, it can be shown by lengthy algebraic manipulation that the exponential term in Eq. (23) is, in fact, the overlap integral $J$ between the acceptor $T_{1} \leftarrow S_{0}$ absorption and the donor $T_{1} \rightarrow S_{0}$ emission if both spectra are approximated by single Gaussian functions with very different widths, i.e., $\sigma_{A}^{2} \gg \sigma_{D}^{2}$.

\section{Nonvertical triplet energy transfer in COT}

The excitation energy dependence of $k_{e}$ given by Eq. (23) can be compared with that obtained from COT experimental energy transfer rate constants ${ }^{28,29}$ by means of the expanded kinetic representation shown in Scheme 2. If all COT triplet-state product molecules decay before being intercepted by the singlet donor (irreversible reaction), the experimental rate constant of the TET process, $k_{\exp }^{\text {en }}$, can be expressed ${ }^{16}$ by 


$$
k_{\exp }^{\mathrm{en}}=\frac{k_{d}}{1+\frac{k_{-d}}{k_{e}}+\frac{k_{-e}}{k_{e}}} .
$$

The probability of reverse transfer $\left(k_{-e}\right)$ from triplet COT to the donor within the collision complex is also likely to be negligible (geminate irreversibility ${ }^{39}$ ), because of the expected very fast relaxation of the triplet energy (see above). Therefore, if the ratio $k_{-e} / k_{e} \ll 1$, Eq. (24) reduces to

$$
k_{\exp }^{\text {en }}=\frac{k_{d}}{1+\frac{k_{-d}}{k_{e}}}=\eta_{e} k_{d}
$$

where $\eta_{e}=k_{e} /\left(k_{e}+k_{-d}\right)$ is the efficiency of the transfer process in the encounter complex. ${ }^{5}$ Thus, when the energy transfer rate constant is larger than that of complex dissociation $\left(k_{-d}\right)$, the reaction becomes controlled by diffusion. ${ }^{5}$ An expression directly comparable to the experimental logarithmic plot of the COT transfer rate constant as a function of the donor energy, ${ }^{28,29}$ can be obtained by replacing $k_{e}$ in Eq. (25) by its value from Eq. (23), to give (in mol units)

$\log k_{\text {exp }}^{\text {en }}=\log k_{d}-\log \left[1+\frac{k_{-d}}{k_{e}^{0}} \exp \left[\frac{1}{R T}\left(\frac{E_{T}^{A}-E_{T}^{D}}{\gamma}\right)^{2}\right]\right]$.

The computed values (see Sec. III) of the COT geometrical distortion parameter, $\gamma=13.5(\mathrm{kcal} / \mathrm{mol})^{1 / 2}$, and triplet vertical excitation energy, $E_{T}^{A}=65.0 \mathrm{kcal} / \mathrm{mol}$, can be introduced in Eq. (26) to give an expression for $\log k_{\exp }^{\text {en }}$ as a function of the excitation energy $E_{T}^{D}$ of the triplet donor:

$\log k_{\mathrm{exp}}^{\mathrm{en}}=\log k_{d}-\log \left[1+\frac{k_{-d}}{k_{e}^{0}} \exp \left[\frac{1}{R T}\left(\frac{65.0-E_{T}^{D}}{13.5}\right)^{2}\right]\right]$.

Figure 3 shows the satisfactory fit of Eq. (27) to the COT energy transfer experimental data ${ }^{28,29}$ for the selected set of donor molecules, for a value of the ratio $k_{-d} / k_{0}^{e}=0.25$. As mentioned earlier, this series of donor molecules have in common the same electronic orbital parentage $\left(\pi, \pi^{*}\right)$ and a rigid molecular frame.

The analysis of the components of the gradient vector yields a detailed description of the geometrical distortions that facilitate the excitation of the triplet COT. In this case (Fig. 4), the largest contribution is from $\mathrm{C}=\mathrm{C}$ stretching modes, which decrease the bond order of the double bonds and increase the $\pi$-electron density at the single $\mathrm{C}-\mathrm{C}$ bonds. The second important contribution is from torsional modes around the four single $\mathrm{C}-\mathrm{C}$ bonds of the molecule. In contrast, the contribution to the gradient vector from torsional modes around double bonds is negligible.

As a result of these concerted motions, the boat $\left(D_{2 d}\right)$ ground-state conformation of COT is deformed, favoring a more planar geometry of the molecule. Interestingly, highlevel computations ${ }^{24}$ of COT and an experimental study of the transition-state spectroscopy of this compound ${ }^{30}$ both predict a flat octagonal $D_{8 h}$ geometry for the COT triplet state.

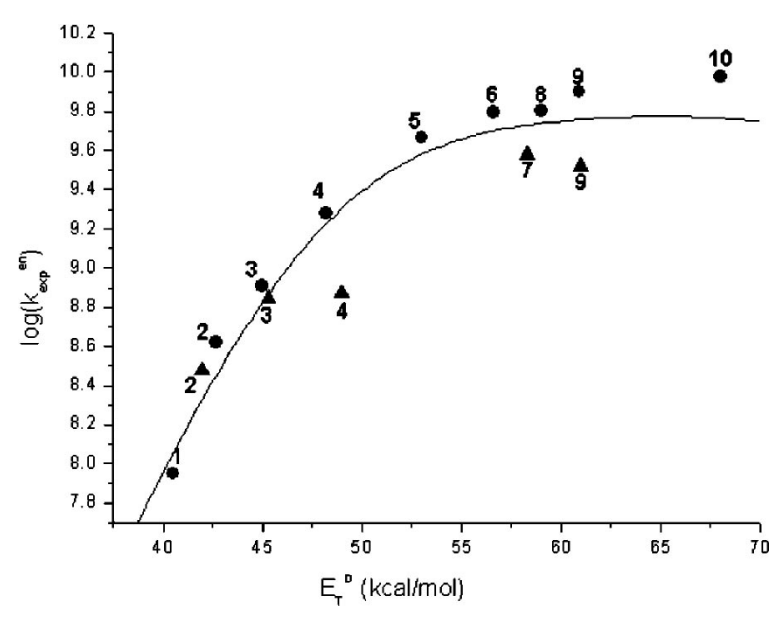

FIG. 3. Plot of the energy transfer rate constant to cyclooctatetraene from a series of $\pi, \pi^{*}$ triplet donors, as a function of the donor energy $E_{T}^{D}$. The experimental data, in benzene solution, were taken from Refs. 28 (O) and 29 (A). The solid line shows the fitting of Eq. (27) in the text to the experimental data, using the theoretical values $13.5(\mathrm{kcal} / \mathrm{mol})^{1 / 2}$ and $65.0 \mathrm{kcal} /$ mol for the distortion parameter $\gamma$ and the COT triplet energy $E_{T}^{A}(300 \mathrm{~K})$, respectively. 1: 9-10, dichloroanthracene, 2: anthracene, 3: acridine, 4: pyrene, 5: fluorenone, 6: chrysene. 7: p-terphenyl, 8: 2-acetophenone, 9: naphtalene, 10: fluorene.

The rms error of the fit in Fig. 3 presents a single minimum (not shown) for a value of 0.25 of the $k_{-d} / k_{0}^{e}$ ratio, the only effective fitting parameter, because that of $k_{d}$ is relatively well defined by the plateau in the experimental data. Since this ratio is unknown "a priori," it was taken here identical for all donor compounds used in the experiment (vide supra) and, therefore, the consistency of the fitting depends on the extent to which the computed $k_{-d} / k_{0}^{e}$ ratio is close to the correct value. Estimated values for the rate constant of the dissociation of the encounter complex $\left(k_{-d}\right)$ in fluid solvents, both from random walk ${ }^{8}$ and more elaborate diffusive arguments, ${ }^{16,39}$ fall in the range of $1-4 \times 10^{10} \mathrm{~s}^{-1}$ (a value of $10^{11} \mathrm{~s}^{-1}$ was derived from macroscopic entropic considerations $\left.{ }^{5}\right)$. Therefore, from the fitting value of $k_{-d} / k_{0}^{e}$ one can estimate a COT preexponential factor $k_{0}^{e} \approx 10^{11} \mathrm{~s}^{-1}$, which is in the same range as those determined directly, ${ }^{4}$ from picosecond-resolved measurements of $k_{e}$, for several aromatic donor/acceptor pairs not structurally related to $\mathrm{COT}$.

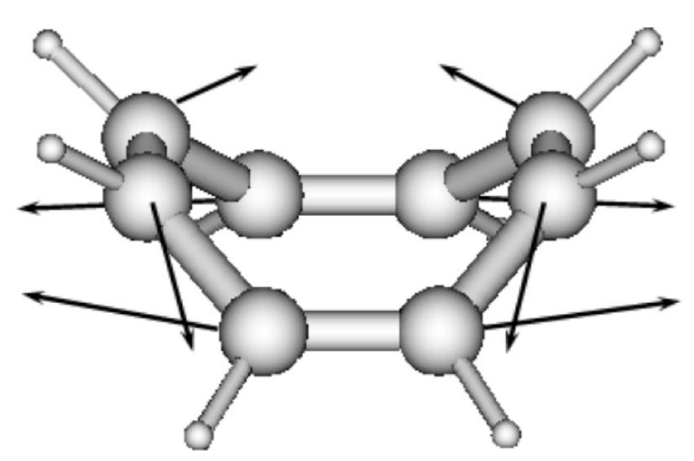

FIG. 4. Pictorial view of the relaxed singlet-ground state of cyclooctatetraene ( $D_{2 d}$ symmetry) illustrating the direction (arrows) of the concerted $\mathrm{C}=\mathrm{C}$ stretching and $\mathrm{C}-\mathrm{C}$ torsional vibrations that decrease the excitation energy to the triplet state. 


\section{METHODS}

The distortion parameter $\gamma$ was determined by calculating the numeric gradient of the $T_{1} \mathrm{PES}$ and the $S_{0}$ numeric vibrational force constants (see Fig. 2) using the multiconfigurational "complete active space" (CAS) Møller-Plesset second-order method CASMP2 $(8,8) / 6-31 \mathrm{~g}^{*}$, as implemented in GAUSSIAN $98 ;{ }^{40}$ the active space in all CAS calculation was taken to be that of $\pi$-symmetry orbitals (the higher four occupied orbitals and the lowest four unoccupied orbitals). The equilibrium geometry of the $S_{0}\left(D_{2 d}\right)$ state was optimized using the analytical gradient at the CASSCF $(8,8) / 6-31 g^{*}$ level of theory. The $E_{T}^{A}$ value was determined theoretically, ${ }^{24}$ in view of the limited experimental data available, using a complete active space secondorder perturbation theory, with a $\operatorname{CASSCF}(8,8) / \mathrm{ANO}$ $\mathrm{C}[4 s 3 p 2 d] / \mathrm{H}[2 s 1 p]$ reference wave function as implemented in MOLCAS 5.0. ${ }^{41,42}$

\section{CONCLUSIONS}

The theoretical model of the triplet energy transfer reaction derived here allows the identification of the physical factors and molecular changes involved in the anomalous nonvertical triplet excitation of flexible acceptors, and provides an interpretation with atomic detail of the molecular motions involved in the process. The present approach may also be of utility in predicting whether the energy transfer rate constant of a specific donor-acceptor pair would show a nonvertical dependence of the rate constant on the donor triplet energy. The application of this theory to existing experimental TET data of COT in solution provides further insight into the origin of the large deviation of the transfer rate constant from Sandros equation. In addition, the groundstate conformational changes responsible for the lowering of the triplet excitation energy could be successfully identified.

\section{ACKNOWLEDGMENTS}

We thank Professor K. Razi Naqvi for crucial comments and Dr. R. E. Dale for helpful suggestions. Work financed by Projects BQU2000/1500, BQU2000/0646, BQU2001/2926 from the Spanish Ministry of Science and Technology (MCyT). L.M.F. is grateful to the Ministry of Education, Culture and Sport (Spain) for a Doctoral Fellowship.

${ }^{1}$ A. Terenin and V. Ermolaev, Trans. Faraday Soc. 52, 1042 (1956)

${ }^{2}$ A. A. Lamola, in Energy Transfer and Organic Photochemistry, edited by P. A. Leemarker (Interscience, New York, 1969), Vol. 14, Chap. II, p. 17.

${ }^{3}$ N. J. Turro, Modern Molecular Photochemistry (Benjamin/Cumming, Menlo Park, CA, 1978).

${ }^{4}$ R. W. Anderson, Jr., R. M. Hochstrasser, H. Lutz, and G. W. Scott, J. Chem. Phys. 61, 2500 (1974).

5 (a) J. Saltiel, P. T. Shannon, O. C. Zafiriou, and A. K. Uriarte, J. Am. Chem. Soc. 102, 2500 (1974); (b) J. Saltiel and B. W. Atwater, Adv. Photochem. 14, 1 (1988).

${ }^{6}$ (a) Th. Förster, Ann. Phys. (Leipzig) 2, 55 (1948); (b) D. L. Dexter, J. Chem. Phys. 21, 836 (1953); (c) K. R. Naqvi and C. Steel, Chem. Phys. Lett. 6, 29 (1970).

${ }^{7}$ K. Sandros, Acta Chem. Scand. (1947-1973) 18, 2355 (1964).

${ }^{8}$ P. J. Wagner and I. Kochevar, J. Am. Chem. Soc. 90, 2232 (1968).

9 (a) J. Saltiel and G. Hammond, J. Am. Chem. Soc. 85, 2515 (1963); (b) G. Hammond and J. Saltiel, ibid. 85, 2516 (1963).
${ }^{10}$ (a) A. Bylina, Chem. Phys. Lett. 1, 509 (1968); (b) S. Yamauchi and T. Azumi, J. Am. Chem. Soc. 95, 2709 (1973); (c) K. Sandros, Acta Chem. Scand. (1947-1973) 27, 3021 (1973); (d) V. Ramamustly and R. S. H. Liu, J. Am. Chem. Soc. 98, 2935 (1976).

${ }^{11}$ J. C. Scaiano and G. G. Wubbels, J. Am. Chem. Soc. 103, 640 (1981).

${ }^{12}$ J. Saltiel, G. R. Marchand, E. Kirkov-Kamiska, W. K. S. Smothers, W. B. Mueller, and J. L. Charlton, J. Am. Chem. Soc. 106, 3144 (1984).

${ }^{13}$ R. A. Caldwell, S. J. Riley, A. A. Gorman, S. P. McNeeney, and D. J. Unett, J. Am. Chem. Soc. 114, 4424 (1992).

${ }^{14}$ J. Catalán and J. Saltiel, J. Phys. Chem. 105, 6273 (2001).

${ }^{15}$ J. Lalevée, X. Allonas, F. Louërat, and J. P. Fouassier, J. Phys. Chem. A 106, 6702 (2002).

${ }^{16}$ (a) V. Balzani and F. Bolleta, J. Am. Chem. Soc. 100, 7404 (1978); (b) V. Balzani, F. Bolleta, and F. Scandola, ibid. 102, 2152 (1980); (c) G. Orlandi, S. Monti, F. Barigetelli, and V. Balzani, Chem. Phys. 52, 313 (1980)

${ }^{17}$ J. Ulstrup and J. Jortner, J. Chem. Phys. 63, 4358 (1975).

${ }^{18}$ M. E. Sigman and G. L. Closs, J. Phys. Chem. 95, 5012 (1991).

${ }^{19}$ (a) A. J. Parola, F. Pina, E. Ferreira, M. Maestri, and V. Balzani, J. Am. Chem. Soc. 118, 11610 (1996); (b) A. Farran and K. D. Deshayes, J. Phys. Chem. 100, 3305 (1996).

${ }^{20}$ C. Serpa, L. G. Arnaut, S. J. Formosinho, and K. R. Naqvi, Photochem. Photobiol. 2, 1 (2003)

${ }^{21}$ S.-T. Levy and S. Speiser, J. Chem. Phys. 96, 3585 (1992).

${ }^{22}$ K. J. Laidler, in Chemical Kinetics (Harper-Collins, New York, 1987), Chap. 4.

${ }^{23}$ (a) L. Landau, Phys. Z. Sowjetunion 2, 46 (1932); (b) C. Zener, Proc. R. Soc. London, Ser. A 137, 696 (); 140, 660 (1933).

${ }^{24}$ L. M. Frutos, O. Castaño, and M. Merchán, J. Phys. Chem. A 107, 5472 (2003).

${ }^{25}$ M. Garavelli, F. Bernardi, A. Cembran, O. Castaño, L. M. Frutos, M. Merchán, and M. Olivucci, J. Am. Chem. Soc. 127, 13770 (2002).

${ }^{26} \mathrm{H}$.-H. Perkampus, in UV-VIS Atlas of Organic Compounds (VCH, Weinheim, 1992), p. 135

${ }^{27}$ R. P. Frueholz and A. Kuppermann, J. Chem. Phys. 69, 3614 (1978).

${ }^{28}$ P. J. Forward, A. A. Gorman, and I. Hamblett, J. Chem. Soc., Chem. Commun. 1993, 250.

${ }^{29}$ T. N. Das and K. I. Priyadarsini, J. Chem. Soc., Faraday Trans. 90, 963 (1994).

${ }^{30}$ P. G. Wenthold, D. A. Hrovat, W. T. Borden, and W. C. Lineberger, Science 272, 1456 (1996).

${ }^{31}$ J. von Neumann and E. P. Wigner, Phys. Z. 30, 467 (1929).

${ }^{32}$ The term nonadiabatic transition is used here in its usual sense, that is, to indicate the transition involving two (or more) interacting PESs.

${ }^{33}$ V. L. Ermolaev, Sov. Phys. Dokl. 6, 600 (1967).

${ }^{34}$ A. A. Gorman, R. L. Beddoes, I. Hamblett, S. P. McNeedey, A. L. Prescott, and D. J. Unett, J. Chem. Soc., Chem. Commun. 1991, 963.

${ }^{35}$ F. Abu-Hasanayn and W. G. Herkstroeter, J. Phys. Chem. A 105, 1214 (2001).

${ }^{36}$ (a) R. A. Marcus, J. Phys. Chem. 24, 966 (1956); (b) Faraday Discuss. 29, 21 (1960); (c) R. A. Marcus, Annu. Rev. Phys. Chem. 15, 155 (1964)

${ }^{37}$ S. J. Formosinho, L. G. Arnaut, and R. Fausto, Prog. React. Kinet. 23, 1 (1998).

${ }^{38}$ M. Bodesheim, M. Schütz, and R. Schmidt, Chem. Phys. Lett. 221, 7 (1994).

${ }^{39}$ B. Nickel, Z. Phys. Chem. (Munich) 178, 25 (1992).

${ }^{40}$ M. J. Frisch, G. W. Trucks, H. B. Schlegel et al., GAUSSIAN 98, Revision A.7, Gaussian, Inc., Pittsburgh, PA (1998).

${ }^{41}$ K. Andersson, M. R. A. Blomberg, M. P. Fülscher et al., MOLCAs, Version 5, Lund University, Sweden (1999).

${ }^{42}$ (a) B. O. Roos, M. P. Fülscher, P.-Å. Malmqvist, M. Merchán, and L. Serrano-Andrés, in Theoretical Studies of Electronic Spectra of Organic Molecules, edited by S. R. Langhoff (Kluwer Academic, Dordrecht, The Netherlands, 1995), p. 357; (b) B. O. Roos, K. Andersson, M. P. Fülscher, P.-A․ Malmqvist, L. Serrano-Andrés, K. Pierloot, and M. Merchán, in Multiconfigurational Perturbation Theory: Applications in Electronic Spectroscopy, edited by I. Prigogine and S. A. Rice (Wiley, New York, 1996), p. 219; (c) M. Merchán, L. Serrano-Andrés, M. P. Fülscher, and B. O. Roos, in Multiconfigurational Perturbation Theory Applied to Excited States of Organic Compounds, edited by K. Hirao (World Scientific, Amsterdam, 1999), Vol. 4, p. 161. 